\title{
Fluorescent silicon carbide as an ultraviolet-to- visible light converter by control of donor to acceptor recombinations
}

\author{
Jianwu Sun, Satoshi Kamiyama, Valdas Jokubavicius, H. Peyre, \\ Rositsa Yakimova, S. Juillaguet and Mikael Syväjärvi
}

\section{Linköping University Post Print}

N.B.: When citing this work, cite the original article.

Original Publication:

Jianwu Sun, Satoshi Kamiyama, Valdas Jokubavicius, H. Peyre, Rositsa Yakimova, S. Juillaguet and Mikael Syväjärvi, Fluorescent silicon carbide as an ultraviolet-to-visible light converter by control of donor to acceptor recombinations, 2012, Journal of Physics D: Applied Physics, (45), , 235107.

http://dx.doi.org/10.1088/0022-3727/45/23/235107

Copyright: Institute of Physics

http://www.iop.org/

Postprint available at: Linköping University Electronic Press

http://urn.kb.se/resolve?urn=urn:nbn:se:liu:diva-77692 


\section{Fluorescent silicon carbide as an ultraviolet-to-visible light converter by control of donor to acceptor recombinations}

J. W. Sun, ${ }^{1, *}$ S. Kamiyama, ${ }^{2}$ V. Jokubavicius, ${ }^{1}$ H. Peyre,${ }^{3}$ R. Yakimova, ${ }^{1}$ S. Juillaguet, ${ }^{3}$ and M. Syväjärvi, ${ }^{1}$

${ }^{1}$ Department of Physics, Chemistry and Biology, Linköping University, 58183 Linköping, Sweden ${ }^{2}$ Department of Materials Science and Engineering, Meijo University, 1-501 Shiogamaguchi, Tenpaku-ku, Nagoya 468-8502, Japan

${ }^{3}$ Université Montpellier 2, Laboratoire Charles Coulomb UMR 5221, F-34095, Montpellier, France 


\begin{abstract}
:
As an alternative to the conventional phosphors in white LEDs, a donor and acceptor codoped fluorescent $6 \mathrm{H}-\mathrm{SiC}$ can be used as an ultraviolet to visible light converter without any need of the rare earth metals. From experimental data we provide an explanation how the light can be obtained at room temperature by a balance of the donors and acceptors. A steady-state recombination rate model is used to demonstrate that the luminescence in fluorescent $\mathrm{SiC}$ can be enhanced by controlling the donor and acceptor doping levels. A doping criterion for optimization of this luminescence is thus proposed.
\end{abstract}

* Email: jianwusun@gmail.com 


\section{Introduction:}

White light-emitting diodes (LEDs) are anticipated to replace the traditional incandescent light bulbs and fluorescent lamps in the near future due to their high-efficacy and large energy-saving in illumination [1,2]. High-efficiency III-nitride LEDs are now commercially available with efficacies surpassing those of the conventional light sources. They have rapidly evolved in white LEDs illumination. For generation of white light emission, three different approaches are developed. These are blue LED with yellow phosphors; an ultraviolet (UV) LED with red, green and blue phosphors; and a device that combines red, green and blue LEDs [2]. The advantage of the blue LED with yellow phosphors is its higher efficacy compared to the other two approaches. Therefore, today's white LEDs are commonly made by a combination of III-nitride based blue LEDs and a coating of yellow phosphors such as cerium-doped yttrium aluminum garnets [1], which are becoming less available since they contain rare-earth metals. However, the main problem for such white LEDs is that the color temperature is too high and the color-rendering index is too low. A real white LED should cover the full spectral range from blue to red.

Recently, an alternative approach has been suggested to realize a true white LED by an all semiconductor integration of a near UV-LED stack on donor and acceptor co-doped $\mathrm{SiC}$ substrates $[3,4]$. Such donor and acceptor doped substrates absorb the near UV light from the GaN-based stack and re-emit visible light via donor-acceptor-pair (DAP) recombinations. Therefore, this wide bandgap semiconductor can be used as fluorescent medium for the white LEDs instead of conventional phosphors. Two successive fluorescent SiC (f-SiC) layers, in which one is doped by nitrogen (N) and aluminum ( $\mathrm{Al}$ ) and another one is doped by $\mathrm{N}$ and boron (B), are used as a substrate for the growth of the III-nitride based near UV-LED stack. The boron is a deeper acceptor than the aluminum. The $\mathrm{N}$ and $\mathrm{B}$ co-doped $\mathrm{f}-\mathrm{SiC}$ emits yelloworange light while the $\mathrm{N}$ and $\mathrm{Al}$ co-doped $\mathrm{f}-\mathrm{SiC}$ emits blue-green light. It has been demonstrated that the CIE chromaticity coordinates of $x$ and $y$ in the $\mathrm{N}$ and B doped SiC are 0.486 and 0.465 while those in the $\mathrm{N}$ and $\mathrm{Al}$ doped $\mathrm{SiC}$ are 0.137 and 0.085 , respectively [3]. Therefore, by a combination of these two spectra, a full-range of visible spectrum similar to the sun-light spectrum can be realized [3]. Moreover, it has been demonstrated that a nitridebased NUV LED stack having the peak wavelength of $385 \mathrm{~nm}$ is grown directly on the $\mathrm{f}-\mathrm{SiC}$ material to make the device [3]. This opens up the way to develop white LEDs with high color rendering index but without any need of the rare-earth-doped phosphors. 
Although $\mathrm{SiC}$ is indirect bandgap semiconductor, it has been demonstrated that $\mathrm{SiC}$ can be used as a light emitting material. Early blue LEDs were produced by $6 \mathrm{H}-\mathrm{SiC}$ p-n homojunctions [5]. The blue light comes from donor acceptor pair recombination between $\mathrm{N}$ donors and $\mathrm{Al}$ acceptors in the p-n homojunctions. Although such devices exhibited only a power efficiency of $\sim 0.05-0.07 \%(25-35 \mu \mathrm{W}$ for a forward current of $20 \mathrm{~mA}$ at $3.2 \mathrm{~V})$ [5], it demonstrates that the suggested approach to get blue light from the recombination between $\mathrm{N}$ donors and $\mathrm{Al}$ acceptors should be realistic. In addition, compared to the electroluminescence from $6 \mathrm{H}-\mathrm{SiC}$ p-n homojunctions, This approach by using the near UV light excitation from the nitride stack enables one to distribute the carriers of a large volume instead of a thin interface of p-n junction at which the injection carrier concentration is too high and nonradiative recombination probability may be also high due to the interface defects. More importantly, it was recently found that $\mathrm{N}$ and $\mathrm{B}$ co-doped $\mathrm{SiC}$ exhibited the high efficient orange-yellow light emission at room temperature $[3,4]$. Furthermore, a prototype of such a warm-white LED made by the integration of nitride based near UV-LED stack on the $\mathrm{N}$ and $\mathrm{B}$ doped f-SiC substrate has been successfully demonstrated in Ref. 3. We believe that if such donor acceptor pair emissions in the f-SiC layers can be controlled by proper doping levels, the integration of the nitrides UV-LED stack on the f-SiC substrates can be anticipated to achieve the white light emission with the high colour rendering index and the stability/reproducibility of colour quality. This would provide an alternative way to achieve real white light without use of rare-earth doped phosphors.

To realize such semiconductor white LEDs, the key issue is to resolve how the emission efficiency in f-SiC layers can be improved by controlling the doping contents. In the previous works $[4,6]$, we reported the observation of room temperature luminescence in $\mathrm{N}$ and $\mathrm{B}$ codoped f-SiC. However, it is still unknown that how the doping levels affects the emission efficiency and what is the doping criterion for optimization of luminescence.

In this work, we focus on the impact of doping levels on the luminescence intensity and quantum efficiency in f-SiC grown by the Fast Sublimation Growth Process (FSGP) [7]. The $\mathrm{N}$ and $\mathrm{B}$ are used as donor and acceptor species, as an example, to demonstrate how the luminescence can be controlled by a balance of donor and acceptor doping levels. As a consequence, a doping criterion for optimization of this luminescence is proposed.

\section{Experimental:}

Thick $6 \mathrm{H}-\mathrm{SiC}$ layers $(50 \sim 80 \mu \mathrm{m})$ were grown on $1.4^{\circ}$ off-axis $6 \mathrm{H}-\mathrm{SiC}(0001)$ substrates by the FSGP. The growth was performed in a high purity graphite crucible at $1750^{\circ} \mathrm{C}$ for one 
hour. $\mathrm{N}$ doping was controlled by adjusting the $\mathrm{N}_{2}$ gas growth pressure. $\mathrm{B}$ doping was achieved by using polycrystalline $\mathrm{SiC}$ source containing different boron concentrations. To determine $\mathrm{N}$ and $\mathrm{B}$ doping concentrations in the epilayers, secondary ion mass spectroscopy (SIMS) measurements were done by a modified Cameca IMS $4 \mathrm{f}$ equipment with an $\mathrm{O}^{2+}$ ion source. Photoluminescence (PL) spectra were collected at room temperature using a frequency doubled Ar+-ion laser for excitation operated at $\lambda=244 \mathrm{~nm}$ with a high excitation power density of $\sim 60 \mathrm{~W} / \mathrm{cm}^{2}$. A Jobin Yvon-Horiba Triax 550 spectrometer, fitted with a 600 grooves/mm grating and a cooled charge coupled device camera, completed the setup. In the previous work [4], internal quantum efficiency (IQE) value of 95\% was estimated by a comparison of integrated photon counts in $\mathrm{GaN}$ at $10 \mathrm{~K}$ with photon counts in $\mathrm{f}-\mathrm{SiC}$ at $250 \mathrm{~K}$, in which the IQE of $\mathrm{GaN}$ at $10 \mathrm{~K}$ is assumed to be $100 \%$. In this work, all IQE values are obtained from the measurements of directly measured external quantum efficiencies (EQEs) by a system with an integrated sphere, an excitation source and a calibrated monochromatic meter combined with a photomultiplier. And we obtained IQE values at a high excitation density of $27 \mathrm{~W} / \mathrm{cm}^{2}$. The excitation source, which is a $355 \mathrm{~nm}$-frequency-tripled YAG CW laser with a beam diameter of $1 \mathrm{~mm}$, was put into the integrated sphere and was led on $\mathrm{f}-\mathrm{SiC}$ samples. A ratio of PL photon number to absorbed excitation photon number provided the EQEs, and they were divided by a theoretical light extraction efficiency to give IQEs. A light extraction value of $6 \%$ from the layers was used for determining the IQE.

\section{Results and discussion:}

Table I shows the growth condition and SIMS results for three series of samples (labeled as A-, B-, C-series) grown by using the source with B concentrations of $5 \times 10^{17}, 1 \times 10^{18}$ and $4 \times 10^{18} \mathrm{~cm}^{-3}$, respectively. At room temperature, these $\mathrm{N}$ and $\mathrm{B}$ co-doped samples exhibit very efficient visible luminescence. Fig. 1 shows, for instance, the picture of yellowish emission from an $\mathrm{N}$ and $\mathrm{B}$ co-doped $6 \mathrm{H}-\mathrm{SiC}$ epilayer under the excitation of $375 \mathrm{~nm}$ from two commercial UV nitrides LEDs. This simple excitation configuration is essentially identical to the designed white LEDs structure. The only difference is that these two UV LEDs are separated for excitation purpose and far away from the f-SiC layers rather than being as a direct integration of a nitride UV-LED stack on f-SiC. Clearly, the fluorescent SiC has efficient emissions at room temperature even under such weak excitation of the UV LEDs.

Photoluminescence (PL) spectra were collected under a saturated excitation power density $\left(\sim 60 \mathrm{~W} / \mathrm{cm}^{2}\right)$ to make most of donors and acceptors essentially non-ionized. Fig. 2 (a) shows the room temperature PL spectra for the three series of samples grown with sources 
containing B concentrations of $5 \times 10^{17}, 1 \times 10^{18}$ and $4 \times 10^{18} \mathrm{~cm}^{-3}$, respectively. As seen in Table I, for each of series, the source used for the growth contains almost constant B concentration but the $\mathrm{N}_{2}$ growth pressure was changed to adjust the $\mathrm{N}$ doping. All samples give a broad emission band centered at $\sim 584 \mathrm{~nm}(2.12 \mathrm{eV})$, which has already been attributed to the N-B DAP emission in $6 \mathrm{H}-\mathrm{SiC}[8,9]$. The photon energy from the DAP transition is given by

$$
E_{D A P}=E_{G}-E_{D}-E_{A}+E_{C}
$$

where $E_{G}, E_{D}$ and $E_{A}$ are the band gap $(3.0 \mathrm{eV}$ for $6 \mathrm{H}-\mathrm{SiC})$, donor, and acceptor binding energies, respectively. The average coulomb energy $E_{C}=\frac{e^{2}}{4 \pi \varepsilon_{0} \varepsilon R}$ can be roughly estimated by letting the pair separation $R=\left(\frac{3}{4 \pi N_{D}}\right)^{\frac{1}{3}}$, with donor concentration $N_{D} \sim 10^{18} \mathrm{~cm}^{-3}$, giving $E_{C} \sim 0.024 \mathrm{eV}$. The $\mathrm{N}$ binding energies were determined to be $0.081 \mathrm{eV}$ for hexagonal site and 0.138 and $0.142 \mathrm{eV}$ for cubic sites in $6 \mathrm{H}-\mathrm{SiC}$ from the infrared absorption measurements [10]. Since the DAP emission in $\mathrm{SiC}$ is usually dominated by its phonon replicas (mainly longitudinal optical phonon line at maximum of the DAP emission band) [8], we also take into account the energy of the longitudinal optical (LO) phonon $E_{L O}=104 \mathrm{meV}$. Assuming that $\mathrm{N}$ binding energy is $\sim 0.1 \mathrm{eV}$, we find the maximum of the DAP emission band at $E_{D A P}-E_{L O}=3.0-0.1-0.7+0.024-0.104=2.12 \mathrm{eV}$, which gives the boron binding energy $\sim 0.7 \mathrm{eV}$. Although both the shallow B level $(0.3,0.35 \mathrm{eV})[9,10]$ and deep B level $(0.58,0.7$, 0.698-0.723 eV) [8-10] have been largely reported by either electrical or optical measurements, there is no observation of the DAP luminescence coming from the shallow B level and all the DAP emissions demonstrate that the B acceptor has the similar binding energy of $\sim 0.7 \mathrm{eV}$ regardless of the polytypes $[8,9,11]$.

From Fig. 2 (b), it is seen that the PL intensity depends not only on the B concentration in the source but also the $\mathrm{N}_{2}$ gas growth pressure. This indicates the PL intensity is related to both $\mathrm{N}$ and B doping concentrations in the epilayers. Table I shows SIMS results of N and B concentrations in three series of $6 \mathrm{H}-\mathrm{SiC}$. Evidently, the increase of $\mathrm{N}_{2}$ gas pressure results in the increase of nitrogen concentration (denoted $[\mathrm{N}]$ ) in the epilayers. For each of series, the boron concentration (denoted [B]) in the epilayers is almost constant, which is around $50 \sim 60 \%$ of the boron concentration in the source. From the combination of PL and SIMS results, it is evident that the PL intensity is largely dependent on the nitrogen and boron doping concentrations. It should be noted that no visible PL can be observed at room 
temperature in samples which have higher boron concentration than nitrogen, namely, p-type, see sample $\mathrm{C}_{1}$ for instance. This can be explained as following: Since the nitrogen donor is much shallower than the boron acceptor, the Bohr radius of nitrogen is much larger than that of boron. Thus for a given donor-acceptor pair, the radiative recombination rate is determined by the Bohr radius of nitrogen [12]. Assuming that all donors and acceptors become neutral at high excitation, the PL intensity can then be explained in terms of the number of the contributing sites and overlap of the electron and hole wave functions [12]. Therefore, there are more radiative recombination centers in the case when the $\mathrm{N}$ concentration is higher than the $\mathrm{B}$ concentration. There could still be some radiative recombination centers in sample $\mathrm{C}_{1}$, while the non radiative recombinations dominate.

To understand how the nitrogen and boron doping influence the luminescence of $\mathrm{f}-\mathrm{SiC}$, we plot the PL intensities of the three series of samples as a function of the difference of nitrogen and boron concentrations (hereafter denoted as $[\mathrm{N}]-[\mathrm{B}]$ ), see Fig. 3. For samples $\mathrm{A}_{1}$ to $\mathrm{A}_{3}$ with a constant boron concentration $[\mathrm{B}]=3 \times 10^{17} \mathrm{~cm}^{-3}$, the PL intensity first increases when $[\mathrm{N}]-[\mathrm{B}]$ is increasing to $5 \times 10^{17} \mathrm{~cm}^{-3}$. However, further increase of $[\mathrm{N}]-[\mathrm{B}]$ to $2.7 \times 10^{18} \mathrm{~cm}^{-3}$, which is almost one order of magnitude higher than the boron concentration [B], does not increase the luminescence intensity (sample $A_{3}$ ). In samples $B_{1}$ and $B_{2}$ with boron doping of $6 \times 10^{17} \mathrm{~cm}^{-3}$, the value of $[\mathrm{N}]-[\mathrm{B}]$ is increasing from $2.4 \times 10^{18}$ to $5.4 \times 10^{18} \mathrm{~cm}^{-3}$ while PL intensities from these samples have no significant difference. However, the intensities are two times higher than those collected on the A-series of samples having lower boron concentration. Notice that in samples $B_{1}$ and $B_{2}$, the value of $[N]-[B]$ is always higher than $[B]$. Finally, in samples $C_{2}$ and $\mathrm{C}_{3}$ (with $[\mathrm{B}]=2 \times 10^{18} \mathrm{~cm}^{-3}$ ), it is clear that the increase of $[\mathrm{N}]-[\mathrm{B}]$ from $5 \times 10^{17}$ to $1.3 \times 10^{18}$ $\mathrm{cm}^{-3}$ results in a significant increase of PL intensity. From these three series of samples, we can tentatively conclude that the increase of the $[\mathrm{N}]-[\mathrm{B}]$ can enhance the PL intensity in the case of $[N]-[B]<[B]$ while PL intensity becomes saturated when $[N]-[B]>[B]$.

To explain the relationship between the PL intensity and the difference of the nitrogen and boron doping concentrations, we consider the steady-state recombination rate via the DAP transition using the model of Aukerman and Millea [13]. In this model, only one donor and one acceptor level are considered. It is assumed that only one transition occurs between electrons captured on donors and holes captured on acceptors and there are no other nonradiative levels in the band gap. Assuming that donors and acceptors become essentially nonionized under the large steady-state excitation, the net recombination rate $U$ via the DAP transition is given by[13] 


$$
U=N_{m} t\left(1+\frac{t+n_{1} C_{n}+p_{1} C_{p}}{n C_{n}+p C_{p}}\right)^{-1},
$$

where $N_{m}$ is the concentration of the minority impurity, $t$ is transition probability of the DAP recombination, $n$ and $p$ are nonequilibrium carriers, $n_{1}=N_{C} e^{\frac{-E_{D}}{k T}}\left(p_{1}=N_{V} e^{\frac{-E_{A}}{k T}}\right)$ is the electron (hole) concentration when Fermi level falls at the donor (acceptor) energy level $E_{D}$ $\left(E_{A}\right), C_{n}\left(C_{p}\right)$ is the capture probability of electrons (holes) by ionized $D^{+} A^{-}$pairs. By applying this model for n-type $\mathrm{SiC}$, we can get $n_{1} \gg>p_{1}$ due to $E_{D}<<E_{A}$. The electron capture probability is given by $C_{n}=\sigma_{n} v_{n}$ with definitions of electron capture cross section $\sigma_{n}$ and thermal velocity $v_{n}$. The capture cross section can be estimated by the Bohr radius $\alpha$, giving $\sigma \sim \pi \alpha^{2}$ [14]. For a rough estimation: one can expect $\frac{\alpha_{D}^{2}}{\alpha_{A}^{2}} \approx \frac{E_{A}^{2}}{E_{D}^{2}}>>1$ [14], where $\alpha_{D}$ ( $\alpha_{A}$ ) is the donor (acceptor) Bohr radius. This leads to a reasonable conclusion that $C_{n} \gg C_{p}$ in our case of n-type $\operatorname{SiC}(n>p)$, hence, $n_{1} C_{n} \gg>p_{1} C_{p}$ and $n C_{n} \gg p C_{p}$. If assuming $t<n_{1} C_{n}$, Eq. (2) reduces to

$$
U=N_{m} t\left(1+\frac{n_{1}}{n}\right)^{-1}
$$

If the nonequilibrium electron concentration $n$ can be roughly estimated by equilibrium electron concentration $n_{0}=\frac{\left(N_{D}-N_{A}\right) N_{C}}{N_{A} g_{D}} e^{\frac{-E_{D}}{k T}}$, Eq. (3) becomes

$$
U=N_{A} t\left(1+\frac{N_{A} g_{D}}{N_{D}-N_{A}}\right)^{-1},
$$

where $N_{D}$ and $N_{A}$ are the donor and acceptor concentrations, $N_{C}$ is effective conductionband state density, $g_{D}$ is the donor degeneracy factor and we use $g_{D}=2$ in SiC.

From Eq. (4), it is seen that the DAP radiative recombination rate in n-type f-SiC will saturate at a constant of $N_{A} t$ when $\left(N_{D}-N_{A}\right)>>2 N_{A}$ while it is significantly increasing with the increase of $\left(N_{D}-N_{A}\right)$ in the case of $\left(N_{D}-N_{A}\right)<2 N_{A}$. By using Eq. (4) and letting $N_{A}=3 \times 10^{17}, 6 \times 10^{17}$, and $2 \times 10^{18} \mathrm{~cm}^{-3}$, respectively, we can fit all three series of samples by using a constant value of the parameter $t$ and this explains very well why the PL intensity is 
increasing with the increase of $[\mathrm{N}]-[\mathrm{B}]$ when $[\mathrm{N}]-[\mathrm{B}]<2[\mathrm{~B}]$ and the saturation occurs gradually in the case of $[\mathrm{N}]-[\mathrm{B}]>2[\mathrm{~B}]$. Notice that the equation $N_{D}-N_{A}=2 N_{A}$, namely, $n_{0}=n_{1}$, means the equilibrium Fermi level falls at donor energy level $E_{D}$. This suggests that the PL intensity in f-SiC significantly increases with an increasing of equilibrium Fermi level $E_{F}^{0}$ until $E_{F}^{0}=E_{D}$ which is due to the increase of $\left(N_{D}-N_{A}\right)$ while the PL intensity gradually saturates when $E_{F}^{0}>E_{D}$. It should be noted that this model does not consider any nonradiative recombination centers. Actually, the higher doping may create some other defects at which carriers may recombine non-radiatively. We have observed that the PL intensity significantly drops down in some samples in which the $\mathrm{N}$ concentration is much higher than $\mathrm{B}$ concentration. This is because in case of $\left(N_{D}-N_{A}\right)>>2 N_{A}$, the radiative recombination rate is saturated while the crystal quality is degenerated due to the higher doping and more nonradiative defects are thus created so that the luminescence is quenched.

To consider the contribution of the non-radiative defects, we plot the internal quantum efficiency (IQE) as a function of the value of [N]-[B] in Fig. 4. In order to compare with PL data, we also use high excitation $\left(27 \mathrm{~W} / \mathrm{cm}^{2}\right)$ for the measurements. As seen in Fig. 4, the IQE shows almost the same trend of the IQE with increasing $[\mathrm{N}]-[\mathrm{B}]$ as that of the PL intensity except sample $\mathrm{B}_{2}$. It is known that

$$
I Q E=\frac{1}{1+\frac{\tau_{R}}{\tau_{N R}}},
$$

in which $\tau_{R}$ and $\tau_{N R}$ are radiative and non-radiative lifetime, respectively. From a comparison of Figs 3 and 4, it is clear that the increase of the IQE (or PL intensity) is due to the increase of the DAP radiative recombination rate (thus decrease of $\tau_{R}$ ) when the value of $[\mathrm{N}]-[\mathrm{B}]$ is increased. However, in case of $\left(N_{D}-N_{A}\right)>>2 N_{A}$, the radiative recombination rate gradually saturates, the further increase of the doping results in more nonradiative recombination channels and thus the $\tau_{N R}$ plays a more important role on the recombination, like indicated by the drop of $I Q E$ in sample $\mathrm{B}_{2}$ having a higher doping. Although the performance of this proof-of-concept material is modest, with IQE efficiencies of $2.5 \%$ at high excitation density of $27 \mathrm{~W} / \mathrm{cm}^{2}$, this work shows the potential path for higher efficiency white LEDs by a proper route of doping. To further increase efficiencies, both the balance between donor and acceptor doping concentrations and control of crystal quality require further investigations. 


\section{Conclusion:}

In conclusion, we demonstrate that an efficient luminescence can be obtained at room temperature in nitrogen and boron co-doped $6 \mathrm{H}-\mathrm{SiC}$. It is found that the PL intensity is increasing with the increase of $[\mathrm{N}]-[\mathrm{B}]$ and gradually saturates when $[\mathrm{N}]-[\mathrm{B}]>2[\mathrm{~B}]$ while the PL intensity will drop down due to the non-radiative defects in the case of $[\mathrm{N}]-[\mathrm{B}]>>2[\mathrm{~B}]$. Thus, the route to optimize the luminescence is that the B concentration should be higher than $2 \times 10^{18} \mathrm{~cm}^{-3}$ and we suggest that a criterion of $\mathrm{N}$ and $\mathrm{B}$ doping is $[\mathrm{N}]-[\mathrm{B}] \sim 2[\mathrm{~B}]$. The IQE observations indicate that the efficiency may be influenced by non-radiative recombination at an increased doping concentration.

\section{Acknowledgements}

This work was supported by Ångpanneföreningen Research Foundation, Richerts Foundation, Swedish Energy Agency, Nordic Energy Research, Swedish Research Council (project No. 2009-5307), Department of the New Energy and Industrial Technology Development Organization (NEDO). 


\section{References:}

[1] Nakamura S and Faso G, “The Blue Laser Diode” (Springer-Verlag, Heidelberg, 1997) 1st edition.

[2] Pimputkar S, Speck J S, DenBaars S P, and Nakamura S 2009 Nature Photonics 3180

[3] Kamiyama S, Iwaya M, Takeuchi T, Akasaki I, Syväjärvi M, and Yakimova R $2011 \mathrm{~J}$. Semiconductors 3213004

[4] Kamiyama S, Maeda T, Nakamura Y, Iwaya M, Amano H, Akasaki I, Kinoshita H, Furusho T, Yoshimoto M, Kimoto T, Suda J, Henry A, Ivanov I G, Bergman J P, Monemar B, Onuma T and Chichibu S F 2006 J. Appl. Phys. 99093108

[5] Edmond J, Kong H, Suvorov A, Waltz D, Carter C Jr 1997 Physica Status Solidi (a) 162 481

[6] Ou Y, Jokubavicius V, Kamiyama S, Liu C, Berg R W, Linnarsson M, Yakimova R, Syväjärvi M, and Ou H 2011 Optical Materials Express 11439

[7] Syväjärvi M and Yakimova R 2011 "Sublimation epitaxial growth of hexagonal and cubic SiC”, Elsevier, chapter in encyclopedia - the Comprehensive Semiconductor Science \& Technology (SEST), Pallab Bhattacharya, Roberto Fornari and Hiroshi Kamimura (Eds), ISBN 978-0-444-53144-5

[8] Ikeda M, Matsunami H and Tanaka T 1980 Phys. Rev. B 222842

[9] Gao Y, I. Soloviev S, and Sudarshan T S 2003 Appl. Phys. Lett. 83905

[10] See a recent detailed review: Lebedev A A 1999 Semiconductors 33107

[11] Kuwabara H, Yamada S, and Tsunekawa S 1976 J. Lumin. 12-13 531

[12] Thomas D G, Hopfield J J, and Augustyniak W M 1965 Phys. Rev. 140 A202

[13] Aukerman L W and Millea M F 1966 Phys. Rev. 148759

[14] Ivanov I G, Magnusson B, and Janzén E 2003 Phys. Rev. B 67165211 


\section{Table and Figure Legends:}

Table I: Growth conditions and SIMS results of nitrogen and boron concentrations in three series of $6 \mathrm{H}-\mathrm{SiC}$ epilayers (A-, B-, C-series).

Figure 1 A picture of efficient yellowish emission from the nitrogen and boron co-doped $6 \mathrm{H}-$ $\mathrm{SiC}$ under the excitation of $375 \mathrm{~nm}$ from two commercial UV LEDs. The sample was grown on one quarter of two inch $6 \mathrm{H}-\mathrm{SiC}$ wafer.

Figure 2 (a) Room temperature PL spectra of three series of samples listed in Table I. (b) PL integrated intensity as a function of $\mathrm{N}_{2}$ gas growth pressure for three series of samples grown with the source containing boron concentration of $5 \times 10^{17}, 1 \times 10^{18}, 4 \times 10^{18} \mathrm{~cm}^{-3}$, respectively.

Figure 3 Room temperature PL integrated intensity as a function of the difference of nitrogen and boron concentrations $([\mathrm{N}]-[\mathrm{B}])$ in the epilayers for three series of samples listed in Table I. Solid lines are the fitting curves according to the Eq. (4) with boron concentration of $3 \times 10^{17}, 6 \times 10^{17}, 2 \times 10^{18} \mathrm{~cm}^{-3}$, respectively. Dashed lines donates the case of $[\mathrm{N}]-[\mathrm{B}]=2[\mathrm{~B}]$ in which the equilibrium Fermi level falls at donor level.

Figure 4 Internal quantum efficiency $(I Q E)$ as a function of the difference of nitrogen and boron doping concentrations $([\mathrm{N}]-[\mathrm{B}])$ for three series of samples with boron doping concentration of $3 \times 10^{17}, 6 \times 10^{17}, 2 \times 10^{18} \mathrm{~cm}^{-3}$, respectively. 
Table I: Growth conditions and SIMS results of nitrogen and boron concentrations in three series of 6H-SiC epilayers (A-, B-, C-series).

\begin{tabular}{ccccccccc}
\hline Sample & $\mathbf{A}_{\mathbf{1}}$ & $\mathbf{A}_{\mathbf{2}}$ & $\mathbf{A}_{\mathbf{3}}$ & $\mathbf{B}_{\mathbf{1}}$ & $\mathbf{B}_{\mathbf{2}}$ & $\mathbf{C}_{\mathbf{1}}$ & $\mathbf{C}_{\mathbf{2}}$ & $\mathbf{C}_{\mathbf{3}}$ \\
\hline $\mathbf{N}_{\mathbf{2}}$ pressure $(\mathbf{m b a r})$ & $1 \times 10^{-5}$ & $1 \times 10^{-2}$ & $1 \times 10^{-1}$ & $1 \times 10^{-2}$ & $5 \times 10^{-1}$ & $1 \times 10^{-2}$ & $1 \times 10^{-1}$ & $5 \times 10^{-1}$ \\
\hline$[\mathbf{B}]$ in the source $\left(\mathbf{c m}^{-3}\right)$ & & $5 \times 10^{17}$ & & & $1 \times 10^{18}$ & & $4 \times 10^{18}$ & \\
\hline$[\mathbf{B}]$ in the epilayer $\left(\mathbf{c m}^{-3}\right)$ & $3 \times 10^{17}$ & $3 \times 10^{17}$ & $3 \times 10^{17}$ & $6 \times 10^{17}$ & $6 \times 10^{17}$ & $2 \times 10^{18}$ & $2 \times 10^{18}$ & $2.7 \times 10^{18}$ \\
\hline$[\mathbf{N}]$ in the epilayer $\left(\mathbf{c m}^{-3}\right)$ & $4 \times 10^{17}$ & $8 \times 10^{17}$ & $3 \times 10^{18}$ & $3 \times 10^{18}$ & $6 \times 10^{18}$ & $1 \times 10^{18}$ & $2.5 \times 10^{18}$ & $4 \times 10^{18}$ \\
\hline Conductive type & $\mathrm{n}$ & $\mathrm{n}$ & $\mathrm{n}$ & $\mathrm{n}$ & $\mathrm{n}$ & $\mathrm{p}$ & $\mathrm{n}$ & $\mathrm{n}$ \\
\hline
\end{tabular}




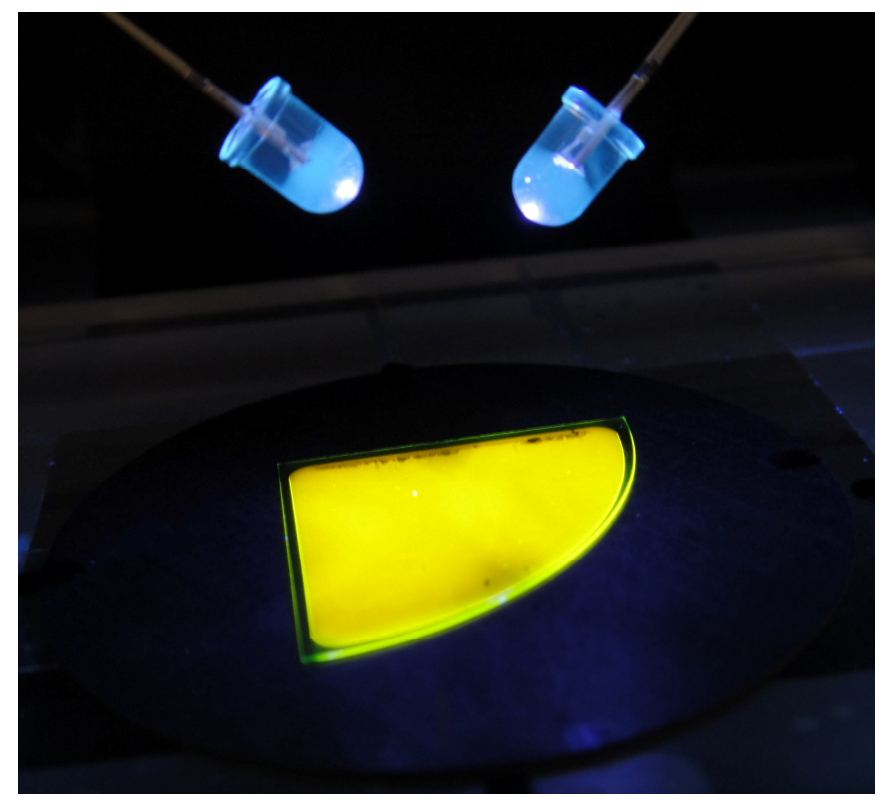

Figure 1 A picture of efficient yellowish emission from the nitrogen and boron co-doped $6 \mathrm{H}-$ $\mathrm{SiC}$ under the excitation of $375 \mathrm{~nm}$ from two commercial UV LEDs. The sample was grown on one quarter of two inch $6 \mathrm{H}-\mathrm{SiC}$ wafer. 

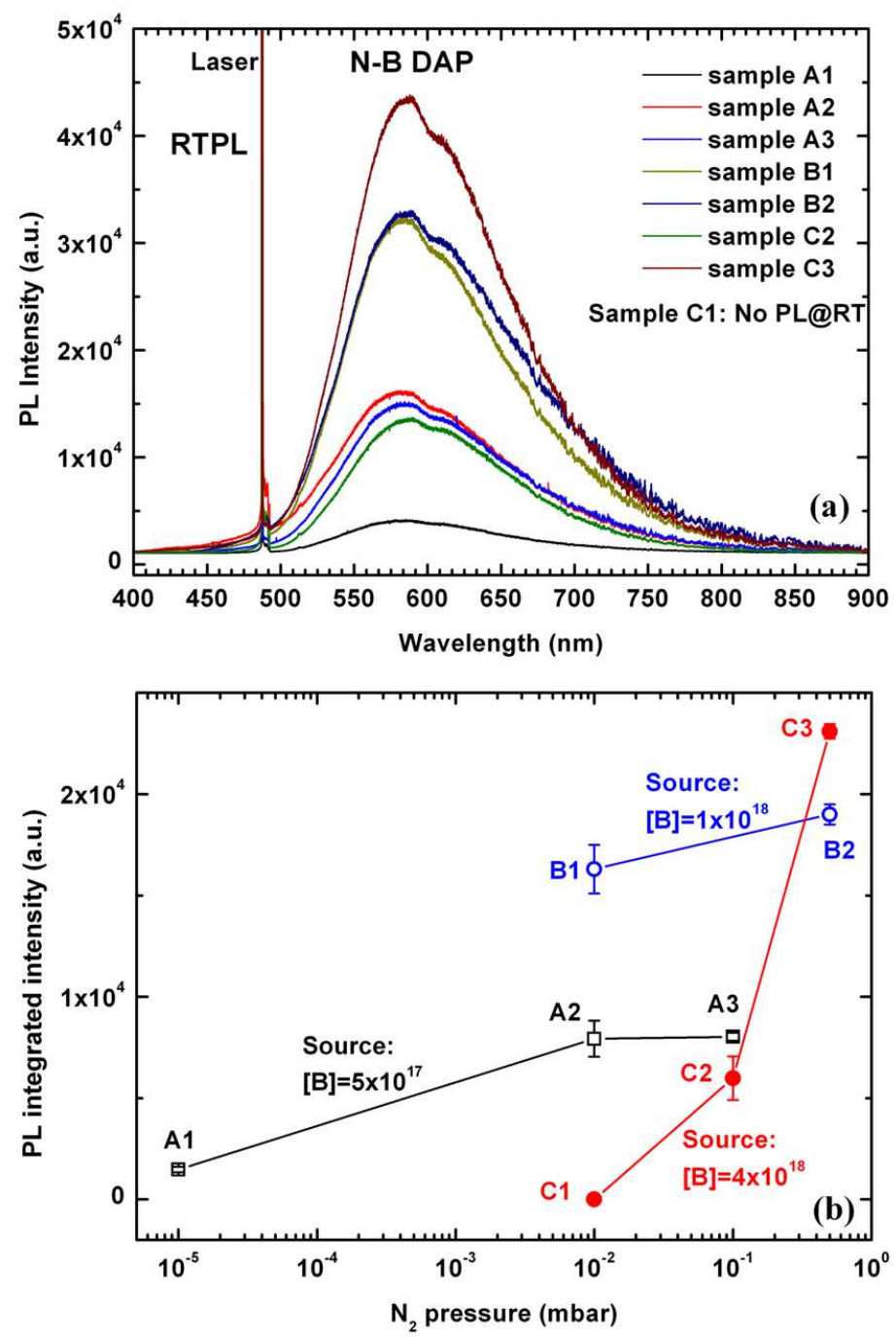

Figure 2 (a) Room temperature PL spectra of three series of samples listed in Table I. (b) PL integrated intensity as a function of $\mathrm{N}_{2}$ gas growth pressure for three series of samples grown with the source containing boron concentration of $5 \times 10^{17}, 1 \times 10^{18}, 4 \times 10^{18} \mathrm{~cm}^{-3}$, respectively. 


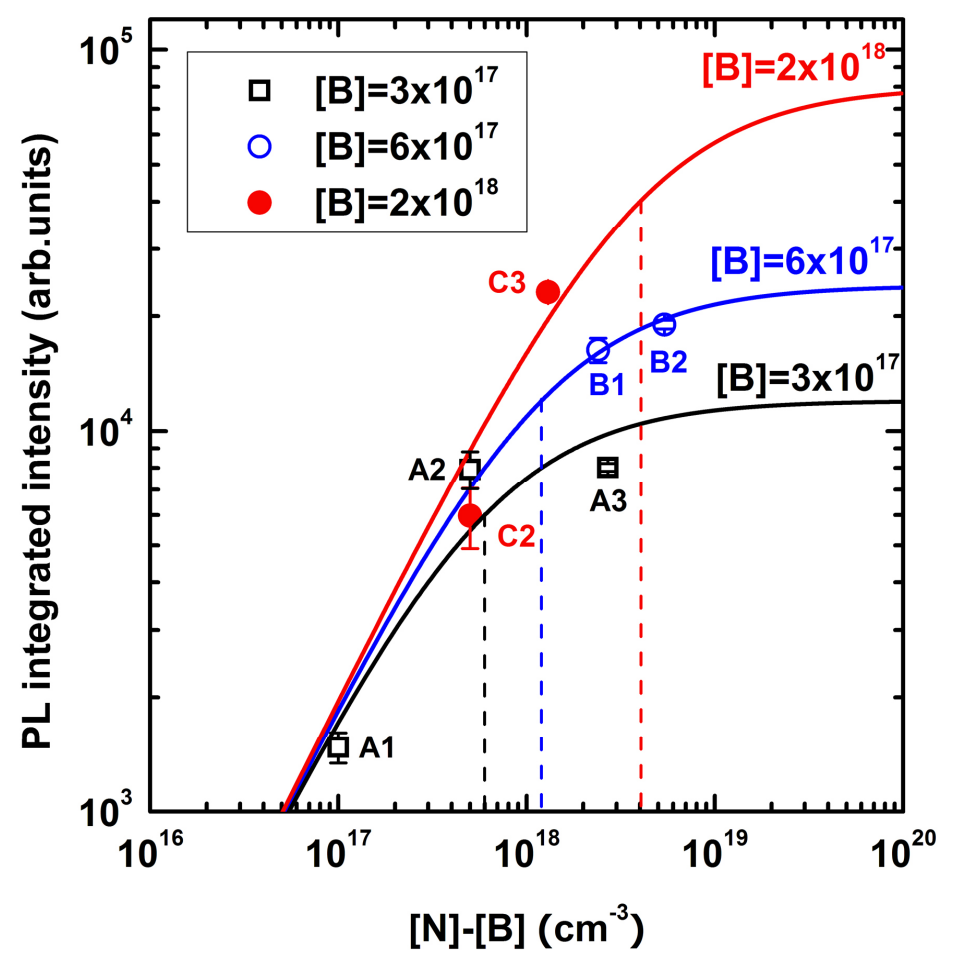

Figure 3 Room temperature PL integrated intensity as a function of the difference of nitrogen and boron concentrations ([N]-[B]) in the epilayers for three series of samples listed in Table I. Solid lines are the fitting curves according to the Eq. (4) with boron concentration of $3 \times 10^{17}, 6 \times 10^{17}, 2 \times 10^{18} \mathrm{~cm}^{-3}$, respectively. Dashed lines donates the case of $[\mathrm{N}]-[\mathrm{B}]=2[\mathrm{~B}]$ in which the equilibrium Fermi level falls at donor level. 


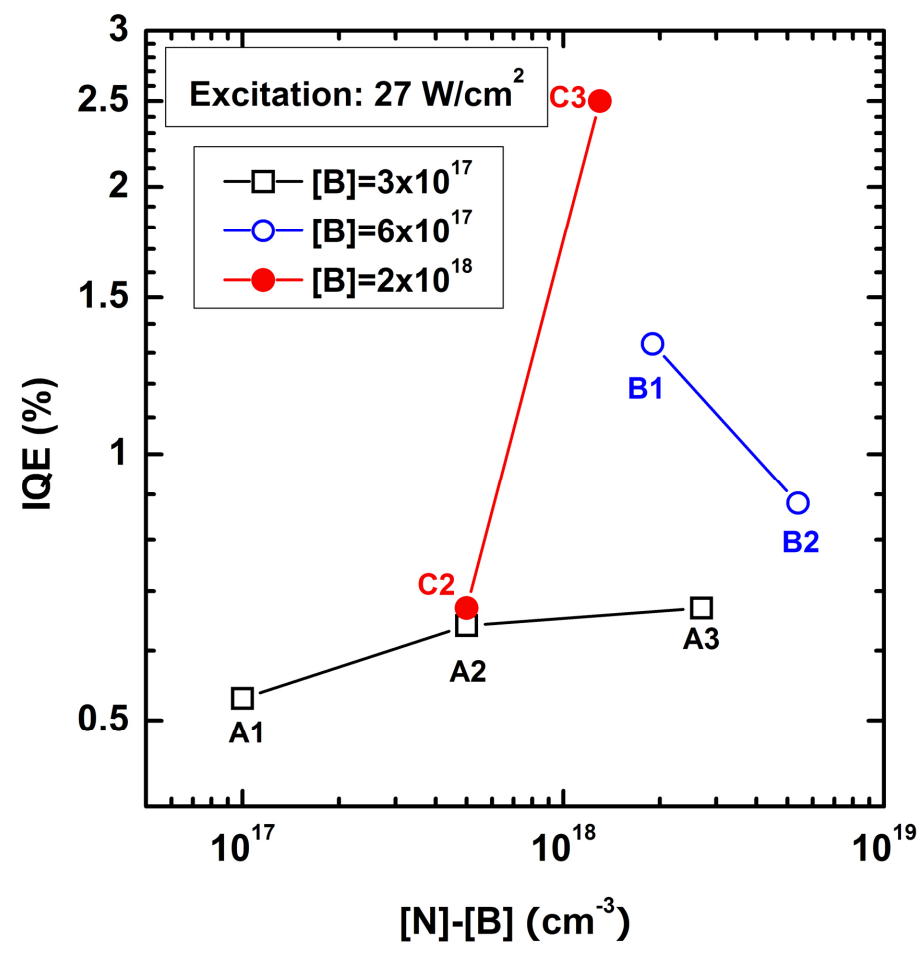

Figure 4 Internal quantum efficiency $(I Q E)$ as a function of the difference of nitrogen and boron doping concentrations $([\mathrm{N}]-[\mathrm{B}])$ for three series of samples with boron doping concentration of $3 \times 10^{17}, 6 \times 10^{17}, 2 \times 10^{18} \mathrm{~cm}^{-3}$, respectively. 